\title{
Politique de la concurrence et intégration des marchés intérieurs : analyse comparative entre les perspectives canadienne et européenne (U.E)
}

\author{
Karounga Diawara
}

\section{Résumé}

L'objet de cet article est d'analyser les objectifs de la politique de la concurrence au Canada et en Europe relativement à l'objectif d'intégration des marchés intérieur. L'auteur montre que les politiques canadienne et européenne en matière de concurrence n’ont pas les mêmes orientations quant à l'objectif d'intégration des marchés intérieurs. Tandis qu'en Europe, la législation antitrust constitue un moyen pour parvenir à l'objectif essentiel d'édification d'un marché intérieur; au Canada, les règles de la concurrence visent d'abord et avant tout à promouvoir l'efficience économique et à lutter contre le gaspillage des ressources économiques rares. Dans la première partie, l'auteur met en exergue cette différence d'orientation en insistant sur les contextes constitutionnels et historiques qui expliquent cette différence. Dans la seconde partie, l'auteur analyse la nature juridique différente des deux regroupements d'États qui constitue le fondement principal de cette différence. L’auteur démontre que la nature économique du Traité CE a facilité, dès le début, une politique européenne de la concurrence tournée vers l'érection d'un marché intérieur ouvert et concurrentiel. Alors qu'au Canada, la nature politique de la fédération n'a pas permis d'orienter la politique de concurrence vers l'intégration des marchés provinciaux. 


\section{Introduction}

En édictant des règles en matière de concurrence, chaque État leur assigne des objectifs donnés. Ces objectifs peuvent être divers et variés allant de la recherche de l'efficience économique à la protection du consommateur en passant par la lutte contre le pouvoir de monopole. Les politiques canadienne et européenne de la concurrence obéissent à cette réalité. Elles ont des fonctions différentes liées au contexte historique et économique ayant présidé à leur apparition et à leur développement. Alors qu’au Canada la législation antitrust date de la fin du $19^{\text {ème }}$ siècle (1889); en Europe, les règles de la concurrence ne sont apparues qu'un demi siècle plus tard à savoir au milieu du $20^{\text {ème } \mathrm{S}}$ avec l'avènement de la construction européenne.

Apparues dans des contextes différents, les règles européenne et canadienne de la concurrence, même si elles partagent quelques similitudes, poursuivent des objectifs différents. Il en est ainsi de l’objectif d'intégration des marchés intérieurs. L’objet de cette étude est de mettre en exergue la différence fondamentale qui a prévalu à l'origine et qui demeure encore aujourd'hui entre les perspectives européenne et canadienne dans la mise en relation entre la politique de la concurrence d'une part et l'objectif de décloisonnement des marchés intérieurs d’autre part.

L'analyse des objectifs de la politique de la concurrence de part et d'autre de l’Atlantique montre que relativement à l’objectif d’intégration des marchés intérieurs, la politique canadienne en matière de concurrence, à la différence de celle européenne, ne vise pas à encourager l'interpénétration des marchés et à censurer toute pratique favorisant le cloisonnement des marchés. En effet, les politiques canadienne et 
européenne en matière de concurrence n’ont pas les mêmes orientations relativement à l’objectif d'intégration des marchés intérieurs. Tandis qu'en Europe, la législation antitrust constitue un moyen pour parvenir à l'objectif essentiel d'édification d'un marché intérieur; au Canada, les règles de la concurrence visent d'abord et avant tout à promouvoir l'efficience économique et à lutter contre le gaspillage des ressources économiques rares.

Ainsi nous montrerons dans la première partie cette différence d’orientation des politiques de la concurrence européenne et canadienne quant à l'objectif de décloisonnement des marchés. Dans cette partie, nous insisterons sur les contextes constitutionnels et historiques qui expliquent cette différence. Dans la seconde partie, nous analyserons les fondements de cette différence d'orientation qui sont attribuables à la nature juridique différente des deux regroupements d’États. Il sera démontré que la nature économique du Traité CE a facilité, dès le début, une politique européenne de la concurrence tournée vers l'érection d’un marché intérieur ouvert et concurrentiel. Alors qu'au Canada, la nature politique de la fédération n’a pas permis d’orienter la politique de concurrence vers l’intégration des marchés provinciaux.

1. La différence d'orientation de la politique de la concurrence quant à l'objectif d'intégration des marchés intérieurs : concurrence-moyen v. concurrencecondition

Cette différence d'orientation des politiques européenne et canadienne de la concurrence est révélée notamment par deux facteurs importants. Le premier est historique et contextuel. Il est relatif à la différence qui a caractérisé l'apparition des politiques de la concurrence en Europe et au Canada (1.1). Ce contexte historique 
différent va rejaillir sur la priorité assignée à la politique de la concurrence. C’est l'opposition entre deux conceptions de la politique de la concurrence : la conception canadienne, à l'image de celle américaine, fait de la concurrence une condition d'organisation du marché; alors que la conception européenne fait de la politique de la concurrence un moyen de parvenir à l'érection d’un marché intérieur sans frontières (1.2.).

\subsection{Différence du contexte d'apparition de la politique de la concurrence au Canada et en Europe}

Le contexte historique qui a vu apparaître les politiques canadienne et européenne en matière de concurrence est très différent et explique la différence d'orientation qui existe quant à l'objectif de création d'un marché d'un marché commun ouvert, concurrentiel et interpénétré.

Au Canada, c'est pour lutter contre les coalitions entre les producteurs visant à fixer les prix et ainsi, exploiter le consommateur qu'une première législation contre les ententes et complots a été élaboré en 1889. Il s'agit de l' l'Acte à l'effet de prévenir et supprimer les coalitions formées pour gêner le commerce ${ }^{1}$. Comme aux U.S.A, cette loi visait à combattre la fixation de prix de la part de certains manufacturiers profitant des barrières tarifaires qui isolaient le marché canadien pour se livrer à des complots (Trebilcock, winter, Collins et Iacobucci 2002, 8-9; Bliss 1973, 180). Cette loi, d'abord

\footnotetext{
${ }^{1}$ S.C 52 Vict. (1889), c. 41
} 
insérée dans le code criminel $^{2}$, allait connaître plusieurs modifications avant d'être remplacée par la loi relative aux enquêtes et les coalitions ${ }^{3}$. Ces deux premières principales législations sur la concurrence avaient pour objectif de protéger le public contre les ententes de fixation de prix (dispositions sur les complots) et contre l'exploitation par les monopoles de la vulnérabilité du consommateur canadien. Comme le constate Rioux: «dans le contexte économique de l'époque, caractérisé par l'accroissement de la taille des entreprises et par la progression de la concentration économique, les deux principales sources d’inquiétude tenaient aux inégalités de pouvoir économique et aux pratiques collusoires des entreprises » (Rioux 2000, 8).

Cependant ces objectifs ainsi assignés aux premières législations canadiennes sur la concurrence n’allaient pas être atteints du fait principalement du niveau de preuve élevée exigé par les tribunaux canadiens pour constater les infractions criminelles à la concurrence. En effet, c'est dans l'affaire Irwing que le sort de ces premières lois allait être scellé : la Cour suprême a exigé une preuve d'atteinte à l’intérêt public qui supposait de démontrer non seulement que la pratique en cause porte atteinte à la concurrence mais aussi qu'elle transgresse l'intérêt public (Cour suprême (C.S) 1978, 408). C’est dans ce contexte que le Canada changea sa politique de la concurrence pour la rendre plus flexible en dépénalisant les dispositions en matière de fusion et d’abus de position dominante.

\footnotetext{
2 article 411

${ }^{3}$ S.R.C 1952, c. 314
} 
Cette réforme importante aboutit à l'adoption en 1986 de la loi sur la concurrence $^{4}$ qui allait donner une nouvelle impulsion à la politique canadienne en matière de concurrence lui permettant de mieux répondre à ses objectifs de protection du public orientée vers la recherche essentielle de l'efficience économique. Cette apparition de la politique de la concurrence au Canada contraste avec l'apparition d'un droit de la concurrence en Europe caractérisée par son application dans le cadre de l'érection d’un marché commun européen.

En Europe, l'apparition de la politique de la concurrence a coïncidé avec la construction européenne. Déjà présentes dans le feu Traité de Paris ${ }^{5}$, les règles de la concurrence allaient être établies, dès l'origine de la fondation de la Communauté économique européenne, par le Traité de Rome. Pour les pères fondateurs européens, il était important de prévoir une politique de concurrence dans une Europe d'après guerre marquée par le corporatisme. Et surtout il fallait démanteler les concentrations d'acier et de charbon existant dans le Ruhr allemand (Monnet 1976, 411). C’est ce qui explique que l’Allemagne a été le premier État membre originaire à disposer d'une politique de concurrence imposée par les alliés.

Mais fondamentalement, l'adoption d'une politique de la concurrence dès l'origine avait pour objectif premier d'encourager la construction d'un marché commun européen sans entrave, ouvert et concurrentiel (Gerber 1998, 347). Comme l’a noté Jean

\footnotetext{
${ }^{4}$ L.R.C (1985), c. C-34

${ }^{5}$ Il s'agit du traité CECA (Communauté Européenne du Charbon et de l'Acier) qui a expiré le 23 juillet 2002
} 
Monnet dans ses mémoires: “...c’était une innovation fondamentale en Europe, et l'importante législation antitrust qui règne sur le marché commun trouve son origine dans ces quelques lignes pour lesquelles je ne regrette pas de m'être battu quatre mois durant” (Monnet 1976, 413).

Ainsi, il était évident dès le départ que la politique de la concurrence devait accompagner l'érection d'un marché intérieur. Ainsi des règles s’appliquant autant aux entreprises privées (article 81 et 82, ex 85 et 86) aux entreprises publiques et aux États membres (art. 86 à 88) allaient être imposées afin d’assurer un régime assurant que la concurrence n’est pas faussée dans le marché commun. Ces règles primaires allaient être complétées en 1989 par une législation portant sur le contrôle des concentrations.

Ce tableau historique d'apparition des politiques européenne et canadienne en matière de concurrence montre clairement la différence de base qui les séparera quant à l’objectif d'interpénétration des marchés. Alors que le droit européen a posé dès le début les jalons d'un tel objectif dans le cadre normal de la construction d'un marché commun; au Canada, l'échec connu par les premières lois antitrust allait entraîner une refondation visant à retrouver l'efficience économique recherchée dès le départ. Dès lors, il s’en suit que les priorités assignées à la politique de la concurrence de part et d'autre de l'Atlantique ne sont pas les mêmes.

\subsection{Différence dans les priorités : Efficience v. intégration des marchés}

L’analyse des priorités établit la différence fondamentale qui sépare les politiques européenne et canadienne de la concurrence relativement à l'objectif d'intégration des marchés. Alors que le canada profite de la réforme en profondeur de sa politique de la 
concurrence survenue d'abord en 1976 puis en 1986 pour affirmer de manière nette son orientation vers l'efficience économique; Dans le même temps, les tribunaux européens dans une interprétation téléologique des règles de la concurrence vont consacrer l'objectif fondamental de création d’un marché commun.

Au Canada, la réforme de 1986 qui a abouti à l’adoption de la présente $L$.C allait préciser de façon claire la priorité de la politique de la concurrence. L’article 1.1 de la L.C mentionne que l'objet de la politique en matière de concurrence vise en premier la recherche, la préservation et le développement de l’efficience économique : « La présente loi a pour objet de préserver et de favoriser la concurrence au Canada dans le but de stimuler l'adaptabilité et l'efficience de l'économie canadienne ». Cet objectif d’efficience économique découle de la recommandation du Conseil économique du Canada (ci-après CEC) qui, dans le rapport provisoire sur la politique de concurrence, a préconisé «pour la politique de concurrence l'adoption d'un seul objectif qui consisterait à améliorer le rendement de l'économie et à éviter le gaspillage des ressources, de façon à accroitre le bien-être » (CEC 1969, 23).

En conséquence, il semble que les règles canadiennes en matière de concurrence, à l'image des U.S.A, ont consacré la théorie de la concurrence-condition. Selon cette théorie, le but des règles en matière de concurrence est de garantir un rendement économique maximum. Le droit antitrust protège la concurrence en elle-même, élément indispensable pour le bon fonctionnement du marché. D’où l'appellation de concurrencecondition, car la concurrence est une condition d'existence d'une bonne économie : « Dans un tel modèle (...), on recherchera la concurrence la plus large possible, et la 
réglementation des comportements anticoncurrentiels visera à l'élimination systématique de toute entente, de tout comportement entrainant une diminution de la concurrence, dès que significative » (Bonassies 51). Dès lors, plus il y a la concurrence, meilleurs seront les rendements. Il ne s’agit donc pas de protéger les concurrents mais la concurrence.

Parallèlement en Europe, les règles de la concurrence sont posées pour éviter qu’aucune nouvelle barrière ne vienne prendre la place de celle que la création du marché unique ferait tomber. L’objectif de décloisonnement des marchés poursuivi par le droit européen de la concurrence est consubstantiel au Traité C.E. En effet, la Cour de Justice (ci-après CJCE) dans une interprétation combinée des articles 2, 3g et 81 CE a ainsi posé l'objectif de création d’un marché intérieur poursuivi par la politique de la concurrence :

...la concurrence non faussée visée aux articles 3.g et 85 [actuellement 81] du Traité C.E.E implique l'existence, sur le marché d'une concurrence efficace, c'est à dire de la dose de concurrence nécessaire pour que soient respectées les exigences fondamentales et atteints les objectifs du Traité et, en particulier, la formation d'un marché unique réalisant des conditions analogues à celle d'un marché intérieur » (nous soulignons) (CJCE, 1977, cons. 20).

Dès lors, la politique de la concurrence vient se joindre et compléter les quatre libertés de circulation (personnes- établissement-, marchandise, services, capitaux) pour l’érection d'un marché intérieur européen sans frontières. Et la politique de la concurrence a largement contribué à cet objectif comme l’illustre la sévérité dont font montre les autorités européennes à l'égard des pratiques qui ont pour effet de cloisonner le marché 
européen ${ }^{6}$ (Ehlermann 1992). Ainsi, avec l'élargissement que vient de connaître l’Union, cet objectif de la politique de la concurrence va contribuer à insuffler cette dynamique constante d’interdiction des pratiques de cloisonnement des marchés.

En définitive, la politique européenne de la concurrence constitue un moyen, parmi d'autres, visant à l'érection d’un marché intérieur. À la différence du Canada, l’U.E a opté pour la théorie de la concurrence-moyen. Selon cette conception, la sauvegarde de la concurrence n’est qu'un moyen pour atteindre des objectifs particuliers. Les effets concurrentiels, mais aussi économiques et sociaux d'un comportement restrictif de la concurrence sont pris en compte (Bonassies 1983, 55). Il pourrait, donc, être concevable de restreindre la concurrence pour parvenir à l'un ou l'autre de ces objectifs. La concurrence n’est pas alors érigée comme un principe sacré et inviolable. Elle peut être restreinte si elle tend à remettre en cause l'intégration européenne. Mais surtout, elle doit être protégée chaque fois que les intérêts du marché intérieur le commandent.

\section{Les fondements de la différence d'orientation : Union politique v. Union économique}

La différence d'orientation des politiques canadienne et européenne en matière de concurrence quant à l’objectif d’intégration des marchés intérieurs trouve son fondement principalement dans la nature juridique différente de ces deux unions étatiques. Alors que

\footnotetext{
${ }^{6}$ Infra point 2.1
} 
l’U.E constitue toujours une Union de nature économique, le Canada est d'abord une union politique. Si paradoxal que cela puisse paraître, alors que l'Europe s'est servi des règles de la concurrence pour en faire un moteur d'intégration économique (2.1) ; au Canada, du fait de la nature politique de la fédération, la politique de la concurrence n’a pas été orientée vers une intégration des marchés provinciaux (2.2.).

\subsection{La politique de la concurrence, moteur de l'intégration européenne}

L’U.E est une organisation supranationale à caractère économique. Le Traité de Rome de 1957 a posé, dès l’origine, les bases de cette union économique. Il a institué, dès le début, un régime assurant que la concurrence n’est pas faussée (article 3g CE). Les célèbres articles 81 et 82 (ancien article 85 et 86 du Traité C.E) ont interdit toute entente ou tout abus de position dominante tendant à créer des obstacles à l'unification des marchés européens. De la même façon le contrôle des aides d’État, l’obligation imposée aux États à ne pas prendre de mesures susceptibles de restreindre la concurrence ainsi que la politique de libéralisation des industries en réseau (télécommunications, gaz, poste, chemin de fer etc.) ont encouragé une interpénétration des marchés européens en un marché intérieur ouvert et concurrentiel.

Dès lors, les pratiques anticoncurrentielles favorisant le cloisonnement de marché à savoir les ententes de fixation des prix et les ententes de répartition de marché ${ }^{7}$ ont été

7 Se répartir les marchés est directement contraire à l’objectif de réalisation d'un marché unique puisque cela aboutit à reconstituer des frontières privées, là où les frontières politiques ont été abolies. C’est l'infraction la plus grave avec les infractions portant sur les prix auxquelles elle est souvent liée, puisque les différences de prix peuvent être maintenues grâce à la répartition des marchés qui empêchent les 
systématiquement et rigoureusement condamnées par les autorités antitrust européennes. En effet, la Cour de justice a rapidement interprété de façon téléologique la législation sur la concurrence dans le sens de renforcer le marché commun conformément à l'objectif du traité CE. C’est ainsi que la Cour a montré son aversion envers les ententes de répartition de marché avec protection territoriale absolue. En effet, selon une jurisprudence constante, la protection territoriale absolue stipulée en faveur du distributeur dans un accord de distribution exclusive et destinée à permettre le contrôle et l'entrave des importations parallèles, aboutit au maintien artificiel de marchés nationaux distincts, contraire au traité, de sorte qu'elle constitue une infraction à l'article 81, paragraphe 1(CJCE 1971, 487;1985, 3933;1990, 261; 1998, 1983; 2004, point 85).

De la même manière, la Commission a développé une politique vigoureuse envers les pratiques anticoncurrentielles de cloisonnement du marché qu'elle qualifie de « cancer à l'économie européenne » (Commission 2002, p. 19). Comme le résume bien Ehlermann, ancien responsable de la politique de la concurrence :

The Commission has from the start pursued a hardline policy under Article 85 against market sharing agreements, cartels related to prices, quotas and investments and collective mutual exclusive dealing arrangement between manufacturers and traders. Selling syndicates have also been viewed unfavourably. Such agreements and arrangements are in direct contradiction to the principle of a single market based on effective competition; logically, it was necessary to eliminate them. The Commission's pratice vis a vis abuses of dominant positions by undertakings has developed along the same lines (Ehlermann 1992, 261).

consommateurs d'acheter les produits les plus intéressants là où ils sont et, par voie de conséquence, de déplacer les parts de marché. 
Et jusqu’à présent, la Commission garde cette même antipathie envers ces infractions graves. En $2001^{8}$ et $2002^{9}$, elle a mené une action d'envergure contre les cartels de prix et de répartition de marché ; lutte matérialisée par la création au sein de la Commission depuis 1998 d'une première unité anticartel et en 2002 d'une seconde unité anticartel. Ces deux unités sont chargées de la détection, la poursuite et la sanction des cartels secrets.

Ainsi, L’expérience européenne montre que les règles de la concurrence sont indispensables pour la création d'un marché commun. En effet, les quatre libertés fondamentales ${ }^{10}$ du marché commun ne sont viables et ne trouvent leur éclosion que dans le cadre d'un marché ouvert et concurrentiel. Les entreprises ou l’État, en adoptant des pratiques restrictives de la concurrence, créent de nouvelles barrières dans l'union et ainsi ralentissent la création d'un espace intérieur sans frontière. C’est pourquoi, la politique de la concurrence constitue l’un des moteurs les plus puissants de l’intégration des marchés européens. Comme le souligne Slot: « the primary objective was market integration. This explains inter alia the emphasis of EC competition policy on the elimination of vertical restraints and export bans. The role of competition law as an instrument of market integration was not limited to the initial stages of the integration process. Competition

\footnotetext{
${ }^{8}$ En 2001, la Commission a adopté dix décisions formelles négatives contre 56 entreprises pour un montant global d'amendes de plus 1,800 milliards d'euros.

${ }^{9}$ En 2002, la Commission a adopté 9 décisions formelles négatives contre 56 entreprises pour un montant global d'amendes de plus 1 milliards d'euros.

${ }^{10}$ Il s'agit de la libre circulation des personnes (1) des biens et des marchandises (2) des capitaux (3) et de la libre prestation de service (4).
} 
policy continued to play an important role for completion of the internal market and beyond » (Slot 2004, 445). Ce qui n'est pas le cas du Canada qui, du fait de sa nature fédérale, n’a pas connu les mêmes options.

\subsection{La nature politique de la fédération canadienne, obstacle à une politique de la concurrence tournée vers l'intégration des marchés provinciaux?}

La nature politique de la fédération canadienne va avoir l'effet inverse de négliger l’importance de la politique de la concurrence dans la constitution d’un marché intérieur canadien intégré. Puisqu’un État fédéral « repose sur l’acceptation d’objectifs communs qui englobent et dépassent les données de l'économie » (Safarian 1974, 13).

L’État fédéral canadien est organisé suivant une répartition des compétences entre le niveau fédéral et le niveau provincial (art. 91 à 95 de la Loi constitutionnelle de 1867). L'une des raisons, qui a fait que la politique canadienne en matière de la concurrence n’a pas favorisé dès le début de l’Union une intégration du commerce interprovincial, est liée au fait que la compétence fédérale en matière de concurrence a d'abord été reconnue seulement au titre du droit criminel. C’est pourquoi, les premières législations canadiennes en matière de concurrence étaient des lois exclusivement criminelles. Et aujourd'hui encore les dispositions interdisant les complots sont encore de nature pénale. Cette situation qui impose un fardeau élevé de la preuve n’a pas permis de lutter efficacement contre les complots anticoncurrentiels entrainant un cloisonnement des marchés interprovinciaux, à savoir les accords de fixation des prix et de répartition des marchés. La reconnaissance de la compétence fédérale en matière de concurrence au titre de ses pouvoirs de réglementation du commerce et des échanges (article 91(2)) n’a pas 
non plus permis de tourner la politique de la concurrence vers l'intégration des marchés provinciaux. En effet, cette reconnaissance est intervenue à un moment où l'intégration des marchés canadiens était déjà accomplie en partie. Dès lors, le législateur canadien a alors posé comme priorité à la politique de la concurrence la recherche de l'efficience économique.

La deuxième raison non moins déterminante est constituée par le fait que la Loi constitutionnelle de 1867 avait déjà posé le fondement l’Union économique canadienne. L’article $121^{11}$ constitue l'expression constitutionnelle du marché commun canadien (Safarian 1972, 19 ; Treblicock 2001, 550). L’article 121 assure la libre circulation des marchandises du crû dans toute la grandeur du Canada sans droits de douanes. Cette disposition, telle qu'elle a été interprétée par les tribunaux canadiens, ne crée guère une union économique intégrée mais seulement une union douanière. En effet, la Cour suprême a décidé dans l’affaire Gold Seal Limited c. Dominion Express Company que ce texte a pour « L’objet réel ...d’interdire l’établissement de droits de douane affectant le commerce interprovincial pour les produits de toutes provinces de l’Union » (C.S 1921, 424).

Ainsi, cette disposition n’a pas permis de créer un marché commun intégré; elle n’a permis d'assurer que le libre mouvement des marchandises, occultant la libre circulation des personnes et des capitaux (Safarian 1972, 21). C’est pourquoi, l'article 6

\footnotetext{
${ }^{11}$ L'article 121 dispose que : « Tous articles du crû, de la provenance ou fabrication de l'une quelconque des provinces seront, à dater de l'Union, admis en franchise dans chacune des autres provinces ».
} 
de la Charte canadienne des droits et libertés allait venir compléter la réalisation d'un marché commun canadien en consacrant la liberté de circulation et d'établissement de tout citoyen canadien à la grandeur de la fédération ${ }^{12}$ (de Mestral et Winter 2001). Néanmoins, il aura fallu attendre 1994, suite à l'échec d'établir une disposition constitutionnelle garantissant un marché commun canadien complet, pour que l'Accord sur le commerce intérieur (ci-après $\mathrm{ACI}$ ) soit signé entre le fédéral et chaque province pour assurer l'érection d'un marché intérieur sans frontière. L’ACI vise à « réduire et éliminer, dans la mesure du possible, les obstacles à la libre circulation des personnes, des produits, des services et des investissements à l'intérieur du Canada, et établir un marché intérieur ouvert, performant et stable ». L’ACI n’a pas une force contraignante et ne peut pas être appliqué par les tribunaux. Il s’agit d'un accord politique tenant compte des particularités du fédéralisme canadien (Trebilcock 2001, 586).

En somme, le Canada n'a pas eu besoin d'utiliser sa politique de la concurrence pour développer un marché interprovincial intégré. La nature politique de l’Union, la criminalisation des règles de la concurrence et l'existence d'autres instruments juridiques lui ont permis de réaliser un marché intérieur intégré sans pour autant recourir à sa politique de la concurrence.

${ }^{12}$ L'article 6 (1) et (2) de la Charte dispose que « 1 . Tout citoyen canadien a le droit de demeurer au Canada, d'y entrer ou d'en sortir. 2. Tout citoyen canadien et toute personne ayant le statut de résident permanent au Canada ont le droit :a) de se déplacer dans tout le pays et d'établir leur résidence dans toute province; b) de gagner leur vie dans toute province » 
Pourtant ce constat peut être déplorable si l'on sait, à l’instar de l'exemple européen, que la politique de la concurrence aurait pu aider à encourager une interpénétration des marchés interprovinciaux canadiens. Et comme l’avait recommandé déjà en 1972 Safarian, la politique de la concurrence peut être utilement utilisée pour l’érection d’un marché intégré :

On comprendra la relation qui existe entre la politique de concurrence et le marché commun en se reportant aux propos sur les avantages qui découlent des échanges. En l'absence de barrières à la mobilité interprovinciale des biens, et dans la mesure où le permettent les coûts de transfert, on a supposé que les producteurs se déplaceraient au-delà de leurs marchés locaux et que les consommateurs pourraient s'approvisionner ailleurs que de leurs sources locales. Celles-ci, en retour, retireraient les avantages indiqués. Si des restrictions particulières sur la production et la répartition empêchent cette mobilité, les avantages provenant des échanges s'évaporeront. (...)Une politique efficace de concurrence est nécessaire à la réalisation des avantages à retirer du marché commun (Safarian 1972, 263-264).

\section{Conclusion}

En définitive, s’il est vrai que l’objectif de décloisonnement des marchés n’est pas une priorité de la politique de la concurrence au Canada du fait de plusieurs facteurs relatés ci-dessus dont le premier tient à la particularité de la nature politique de la fédération canadienne. Nous avons vu par contre que la politique européenne en matière de concurrence a largement contribué à créer un marché intérieur européen intégré.

Malgré cette opposition de taille due à des facteurs historico-politiques et économiques, les règles canadiennes en matière de concurrence, à l'instar des règles européennes, contiennent des dispositions susceptibles de combattre certaines pratiques favorisant le cloisonnement des marchés. Mais, il est vrai, que c’est dans l’abnégation du traitement de ces interdictions que se trouve toute la différence. Nous avons vu que les 
autorités antitrust européennes ne tolèrent aucunement les pratiques tendant à empêcher l’interpénétration des marchés. Au Canada, les dispositions de principe existent mais les autorités de concurrence ne font pas de la lutte contre de telles pratiques une priorité. C’est là où réside toute la différence.

Pourtant, le cadre juridique de l'interdiction des comportements encourageant le cloisonnement des marchés canadiens existe bien dans la L.C. Il en est ainsi, aussi bien, des pratiques anticoncurrentielles ${ }^{13}$ que des pratiques restrictives de concurrence ${ }^{14}$. Ainsi donc, le droit canadien de la concurrence réprime en principe les pratiques tendant à cloisonner les marchés, à savoir les pratiques de fixation des prix et les pratiques de répartition des marchés.

\footnotetext{
${ }^{13}$ Il s'agit principalement des complots restrictifs de concurrence interdits par article 45 de la L.C, de l'abus de position dominante interdite par l'article 79 et des fusions régies par les articles 91 et s. de la L.C

${ }^{14}$ Il s'agit notamment des articles 51 interdisant la discrimination par les prix; 61 (1) a) prohibant le maintien de prix; et surtout l'article 77 qui censure dans certaines conditions l'exclusivité, la vente liée et la limitation de marché.
} 


\section{Bibliographie}

Anderson R.D. et Khosla S. D., La politique de concurrence en tant que dimension de la politique économique: une analyse comparative, Industrie canada, Document hors série n7, Ottawa, 1995

Bliss M, « Another Antitrust Tradition: Canadian anti-Combines Policy, 1889-1910”, 47 Business History Review (1973), 177-88

Bonassies Pierre, « Les fondements du droit communautaire de la concurrence : la théorie de la concurrence-moyen », dans Études dédiées à Alex Weill, Paris, Dalloz-Litec, 1983, 51-68

CJCE, Deutsche Grammophon c. Metro SB, C-78/70, Rec. 1971, p.487

CJCE, ETA c. DK Investment, C-31/85, Rec. 1985, p.3933

CJCE, Javico c. Yves Saint Laurent Parfums, C-306/96, Rec. 1998, p.I-1983

CJCE, Tipp-Ex c. Commission, C-279/87, Rec. 1990, p.I-261

Cour suprême, Gold Seal Limited v. Dominion Express Company, (1921), 62 S.C.R. 424

Cour suprême, R. c. Irwing 1978 R.C.S, 408

Ehlermann Claus-Dieter, « The contribution of EC competition policy to the single market », 29 Common Market law Review (1992), 257-282

Gerber David J., Law and competition in twentieth century Europe: protecting Prometheus, Clarendon Press, Oxford (1998)

Goldman Calvin S. et Kissack Joel T., "The role of Competition Policy in Canada's Industrial Policy”, 19 Can.-U.S. Law Journal (1993), 105-130

Gorecki Paul K. et W.T. Stanbury, The Objectives of Canadian Competition Policy 18881983, Montréal, Institut de recherche politique, 1984

Green C, "Canadian Competition Policy: Past and present”, dans Miren A. Letemendia (dir) Les politiques de concurrence de la Communauté économique européenne et du Canada, Montréal, Centre de droit privé et comparé, 1983, 39-66

Khemani R.S. and W.T. Stanbury (dir), Canadian competition law and policy at the centenary, Halifax, Institute for Research on Public Policy, 1991

Khemani R.S. and W.T. Stanbury (dir), Historical perspectives on Canadian competition 
policy, Halifax, Institute for Research on Public Policy, 1991

De Mestral A. and Winter J.,’Mobility Rights in the Union European and Canada”, 46 McGill L. J. (2001), 979-1009

Monnet Jean, Mémoires (Fayard, Paris, 1976)

Rioux Michèle, « le rôle stratégique de la politique de la concurrence du Canada », Cahier de recherche GRIC UQAM 2000-09, juillet 2000 (en ligne : http://www.unites.uqam.ca/gric/pdf/09-2000-rioux.pdf consulté le 20-04-2006)

Safarian A.E, Le fédéralisme canadien et l’intégration économique, Ottawa, Information Canada, 1974

Slot Piet Jan, “a view from the mountain: 40 years of developments in E.C competition law” (41 Common Law Market Review, 443-473, 2004)

T.P.I, JCB Service c. Commission, T-67/01, Rec. 2004, p. II 49

Trebilcock Michael et al., The law and economics of Canadian competition policy, Toronto, University of Toronto Press, 2002.

Trebilcock Michael J.,” The Supreme Court and Strengthening the Conditions for Effective Competition in the Canadian Economy”, 80 La revue du barreau canadien (2001), 543 